\title{
Z-frenuloplasty: A Better Way to 'Untangle' Lip and Tongue Ties
}

\author{
Karishma Dusara*, Avan Mohammed, and Nasser Ahmed Nasser \\ Whipps Cross University Hospital, Barts Health NHS Trust, London, UK
}

Received: November 20, 2013, Accepted: January 14, 2014, Published: January 17, 2014

"Corresponding author: Karishma Dusara, Department of Oral Surgery \& Maxillofacial surgery, Whipps Cross University Hospital, Barts Health NHS Trust, London, UK, Email: karishma.dusara@bartshealth.nhs.uk

\begin{abstract}
Introduction: An abnormal frenulum can cause aesthetic and functional problems such as a midline diastema, localized gingival recession, loss of sulcus depth and ankyloglossia. This may lead to problems with speech articulation and swallowing. The aim of this article was to highlight the use of a novel surgical technique used for the treatment of hypertrophic frenula known as Z-Frenuloplasty.

Method: This technical note outlines the indications and procedure involved in performing a Z-Frenuloplasty. Incisions are made to raise flaps which are later repositioned in a 'Z-plasty' flap closure. In the case of maxillary diastema, the pre - maxilla scar tissue is excised and the site is left to granulate and heal. Sutures are removed a week later.
\end{abstract}

Results: Z-Frenuloplasty helps to release scar contracture and relieve soft tissue tension. The appearance of a scar is also improved with this technique as it helps to both relax and re - align the tissues. This procedure can also be used to position a short frenulum more apically for better aesthetic and functional results.

Conclusion: The appropriate management of hypertrophic frenula can be challenging but with the use of the Z-Frenuloplasty technique, it has been shown that favorable results can be achieved with improved aesthetic and functional outcomes.

Keywords: Z-Frenuloplasty, Frenulum, Frenectomy, Diastema, Ankyloglossia, Z-plasty

\section{Introduction}

The function of a maxillary frenulum is to provide stability to the upper lip [1]. It consists of an attachment of thin fibrous tissue connecting the upper lip to the gingival tissue between the central incisors. Hyperplastic frenula arise when there is a thick fibrous tissue attachment between the upper incisors and are often associated with a midline diastema. It can also result in periodontal and speech problems $[1,7]$.

A lingual frenulum is a thick fibrous attachment under the tongue, which stabilizes tongue movement [1]. A congenital condition known as ankyloglossia (tongue tie) arises as a result of a short and tight lingual frenulum. As a result of limited tongue mobility, ankylogossia can cause difficulty in speech articulation, dyspnea, dysarthria and problems with breastfeeding in babies
$[3,9,10]$. This condition can be self-limiting and most cases resolve spontaneously.

Surgery should be considered at any age if there are speech or feeding difficulties [11]. Several surgical procedures have been implemented to revise abnormal frenula. These include excision, Millers technique, V-Y plasty and Z-plasty [2]. However, frenectomies commonly fail due to a high risk of reoccurrence and hypertrophic scarring $[1,2]$. The risk of failure can be reduced by using a technique known as Z-Frenuloplasty, which is a soft tissue surgery used to lengthen a frenulum. It works best when used for hypertrophic thick frenula with a low insertion and a shallow sulcus. Although this procedure can be carried out under local anaesthetic, general anaesthesia will be required for young children. Patients consented for this procedure are warned to expect mild discomfort, post operative pain, bleeding, swelling and infection of the surgical site.

This technical note aims to outline the technique involved in performing the Z-Frenuloplasty for abnormally positioned labial and lingual frenula.

\section{The Z-Frenuloplasty Technique}

\section{Maxillary labial frenulum}

A surgical marker pen is used to outline the 'Z-shape' to enable the clinician to raise the Z-plasty flap efficiently (Figure 1). Local anesthetic infiltrations are given locally around the frenulum and palatally using 2 cartridges of $2 \%$ lignocaine with 1:80000 adrenaline. A central vertical incision is made with a \#15 blade at the frenulum. The incision is made at the most inferior

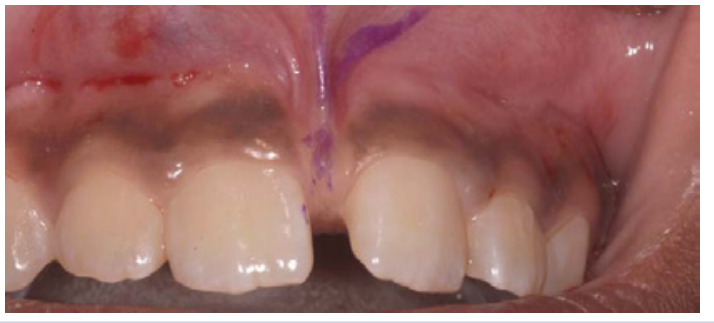

Figure 1: A Diastema caused by a labial frenum in a 14 year old. 
part of the frenulum in an upwards direction (Figure 2). The pre - maxilla scar tissue is excised and the site is left to granulate and heal (Figure 3).

Incisions are then made to create the 'Z-shape' triangular flap (Figure 4). The blade is rotated 90 degrees and a horizontal incision is made in the labial mucosa, starting from the top of the frenulum and then extending outwards. This is approximately $1-2 \mathrm{~cm}$ in length and it creates the triangular flap. Another identical triangular flap is raised on the contra lateral side but at the base of the vertical incision which gives the incision the' $\mathrm{Z}$ - shape'. The flaps are then rotated and repositioned to achieve closure (Figure 5). Resorbable sutures are placed and in this case Vicryl Rapide 4.0 was used (Figure 6)

\section{Lingual frenulum}

The Z-Frenuloplasty technique for a lingual frenulum is very similar to the labial frenulum as described above. However, greater care must be taken to prevent the development of a sublingual haematoma.

Local anesthetic infiltrations are given locally around the frenulum using 1 cartridge of $2 \%$ lignocaine with 1:80000 adrenaline. A black silk suture is placed through the tip of the tongue to allow the surgeon to retract the tongue and hold it in place. A vertical incision is made on the lingual frenulum (Figure7). Two horizontal incisions made at 90 degrees to the vertical incision (Figure 8). The two rectangular flaps are transposed in a Z-plasty manner (Figure 9). Repositioned flaps are sutured in place. In this case a prolene suture was used instead of resorbable sutures due to surgeon preference (Figure 10).

\section{Discussion}

After the above procedures, patients were discharged and

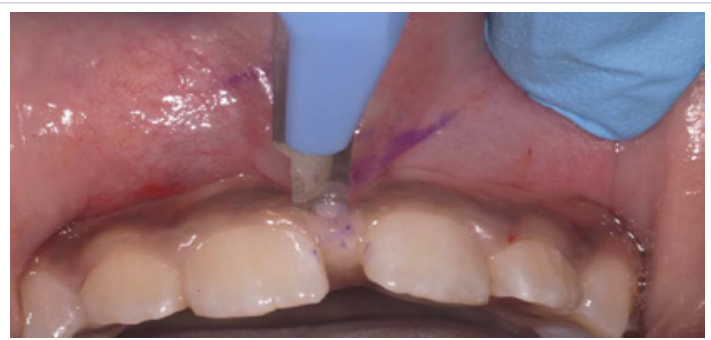

Figure 2: First incision made at the base of the frenum.

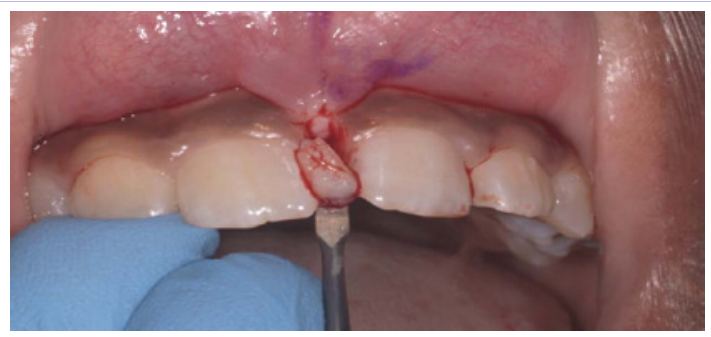

Figure 3: Removal of pre maxilla scar tissue.

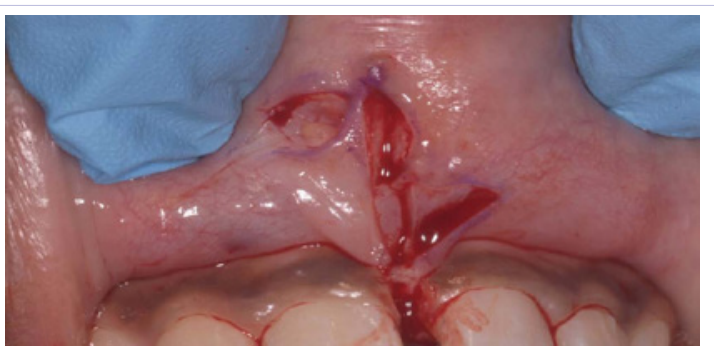

Figure 4: Two triangular flaps are made following the incision (Zshape).

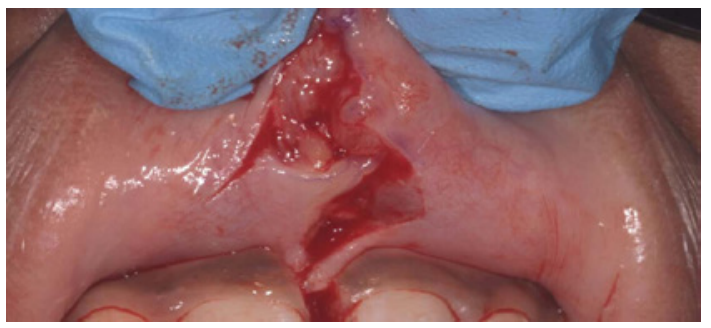

Figure 5: Flaps are repositioned prior to closure.

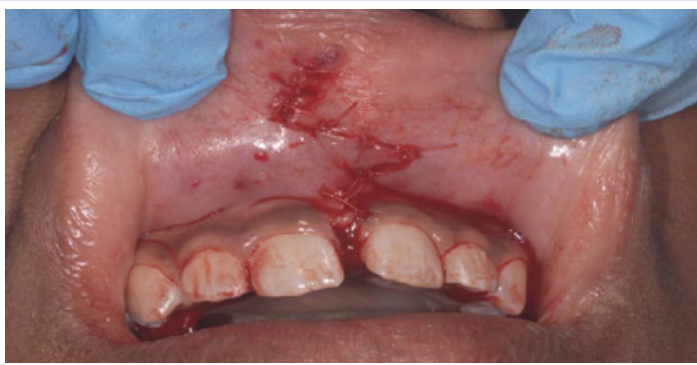

Figure 6: Closure of flaps.

post-operative instructions were given. Patients were asked to maintain a soft diet for a week, take analgesics if necessary and to maintain good oral hygiene. No antibiotics were prescribed. All the patients were seen a week later for a review. Those with orthodontic treatment plans were discharged back to their orthodontists.

By using the Z-Frenuloplasty technique and by repositioning of the flap in this manner, we have found that this results in less soft tissue tension, lengthening of the lip, minimal scarring and improved lip or tongue function. With a co-operative patient this technique can easily be performed under local anaesthetic and unlike other techniques there is no relapse of the Frenulum making it a very reliable treatment.

A study by Heller et al 2005 [3] compared the treatment outcome for Z-frenuloplasty and the traditional horizontal to vertical frenuloplasty in the management of Ankylogossia. A total of 16 patients were recruited to the study; 11 patients underwent a Z-frenulplasty and 5 patients had their Ankylogossia corrected with the horizontal to vertical frenuloplasty. The pre and post treatment analysis included the frenulum length, length of tongue protrusion and speech. The results revealed 
the Z-frenuloplasty to be a more superior technique, showing an improvement of Frenulum length and tongue protrusion of 37.5 $\pm 13.5 \mathrm{~mm}(\mathrm{P}<0.001)$ compared to the other method at $36.2 \pm 7.6$ $\mathrm{mm}(\mathrm{P}<0.001)$, respectively. Furthermore, it was also found that $91 \%$ of the patients who underwent the Z-frenuloplasty showed improvement in speech compared to only $40 \%$ in those who underwent the horizontal-to-vertical frenuloplasty.

The literature reports other surgical methods used for the treatment of hypertrophic frenulums such as V-Y frenuloplasty [5] which involves making $\mathrm{V}$-shaped incision under the frenal attachment. The flap is raised and repositioned into a Y- shape and sutured into place. The Millers technique [4-6] is used for removing upper midline diastemas only. The frenulum is excised completely exposing bone. A pedicle graft is taken from the palate and sutured into place across the midline. A periodontal dressing is placed and the patient is reviewed a week later. Electrosurgery [6] and lasers has also been advocated for frenectomies for patients with bleeding disorders.

\section{Conclusion}

The current evidence although limited does suggest that the Z-Frenuloplasty is a superior technique when compared to

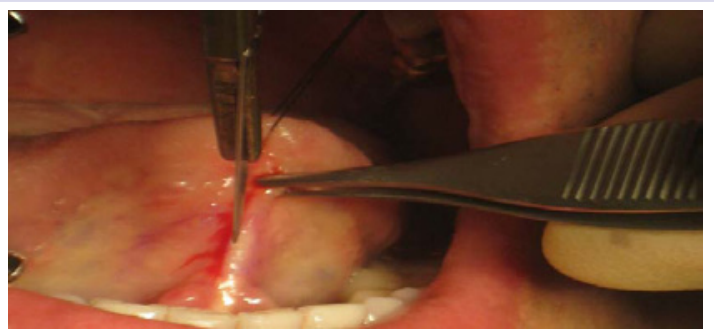

Figure 7: Tongue retraction and a vertical incision is made through the frenum.

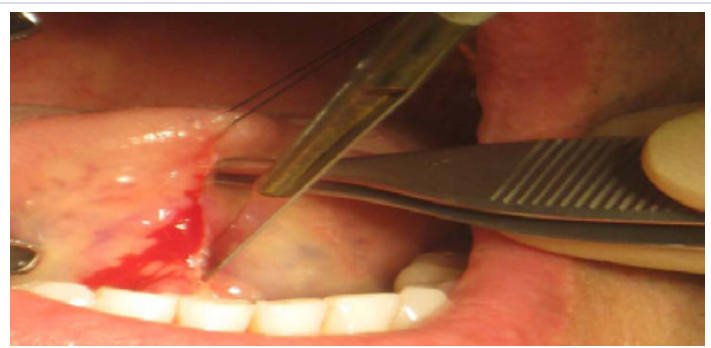

Figure 8: Z- shaped triangular flap incision.

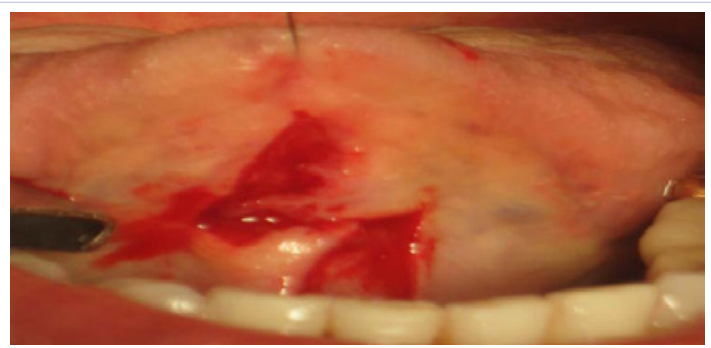

Figure 9: Triangular flaps repositioned.

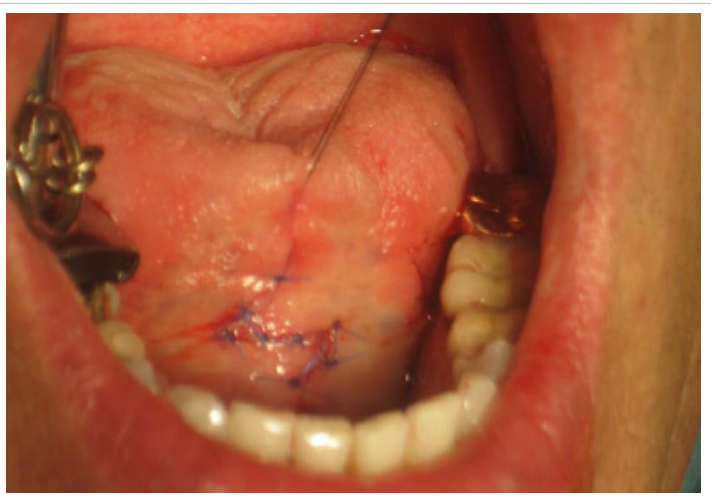

Figure 10: Sutures used to close wound.

other techniques [8]. However, care must be taken as with any surgical procedure. Extensive division of a lingual frenulum can damage the blood vessels in the floor of the mouth. Rare cases of sublingual haematoma and also Ludwigs angina have been reported in the literature [3]. It is therefore important that this procedure is only carried out by an experienced clinician.

Overall the Z-Frenuloplasty procedure is considered to be safe, cost effective and results in better functional and aesthetic appearance. This procedure allows for tissue healing by primary intentions; increasing recovery and reducing the risk of tissue contractures. However due to the limited evidence currently available on this procedure, further research is proposed in the field to compare the different surgical frenectomy procedures [12].

\section{References}

1. Huang WJ, Creath CJ (1995) The midline diastema: a review of its etiology and treatment. Pediatr Dent 17 (3): 171-9

2. Puig JR, Lefebvre E, Landat F (1977) [Z-plasty technic, applied to hypertrophy of the upper labial frenum]. Rev Stomatol Chir Maxillofac 78(5):351-6

3. Heller J, Gabbay J, O’Hara C, Heller M, Bradley JP (2005) Improved ankyloglossia correction with four-flap Z-frenuloplasty. Ann Plast Surg 54(6):623-8.

4. Jhaveri H (2006) The Aberrant Frenum. Dr. PD Miller the father of periodontal plastic surgery 29-34.

5. Kahnberg KE (1977) Frenum surgery. I.A comparison of three surgical methods. Int J Oral Surg 6(6): 328-33.

6. Devishree, Gujjari SK, Shubhashini PV (2012) Frenectomy: A review with the reports of surgical techniques. J Clin Diagn Res 6(9):1587-92

7. Bagga, Sukhchain, Bhat, K Mahalinga, Bhat, G Subraya, Thomas, Betsy S (2006) Esthetic management of the upper labial frenum: a novel frenectomy technique Quintessence Int 37(10): 819-23

8. Morselli, Paolo, Vecchiet, Federico, Marini, Ida (1999) Frenuloplasty by means of a triangular flap. Oral Surg Oral Med Oral Pathol Oral Radiol Endod 87(2): 142-144

9. Mcbride Craig (2005) Tongue-tie. Journal of paediatrics and child health 41(5-6): 242

10. Brinkmann S, Reilly S, Meara JG (2004) Management of tongue-tie in 
children: a survey of paediatric surgeons in Australia. J Paediatr Child Health 40(11): 600-605

11. Marchesan IQ, Martinelli RL, Gusmao RJ (2012) Lingual frenulum: changes after frenectomy . J Soc Bras Fonoaudiol 24(4): 409-12

12.Suter VG, Bornstein MM (2009) Ankyloglossia: facts and myths in diagnosis and treatment. J Periodontol 80(8): 1204-1219. 